\title{
Retaining Precision at Low-dose and High-speed STEM Imaging Conditions
}

Tiarnan Mullarkey ${ }^{1}$, Clive Downing ${ }^{2}$ and Lewys Jones ${ }^{3}$

${ }^{1}$ Centre for Doctoral Training in the Advanced Characterisation of Materials. School of Physics, Trinity College Dublin, Dublin 2, Ireland, Dublin, Dublin, Ireland, ${ }^{2}$ Advanced Microscopy Laboratory, Centre for Research on Adaptive Nanostructures and Nanodevices (CRANN), Dublin 2, Ireland, Dublin, Dublin, Ireland, ${ }^{3}$ Trinity College Dublin, Dublin, Dublin, Ireland

With a modern aberration corrected scanning transmission electron microscope (STEM) it is possible to reach the instrumental resolution when working with large electron-doses and, quite importantly, a sample able to withstand them. However, when working with beam-sensitive samples we find instead that a 'doselimited resolution' becomes the limiting factor [1], [2]. Beyond just resolution and considering information precision instead of image-resolution we again find the electron-dose to be crucial [3].

For minimising electron-dose and/or dose-rate there are many avenues to explore; rastering the beam at higher speeds, lowering the beam current, and using a shorter flyback time (flyback dose causes damage but yields no information). Each of these approaches are readily available to the operator with no need for additional expensive hardware, but regrettably they each also introduce different unacceptable artefacts to the final image.

These artefacts can include:

- the erroneous streaking of signal across multiple pixels when dwell-time is shorter than the scintillator decay time (figure 1),

- afterglow leading to a non-zero offset on the detector output leading to Poisson noise, even in areas of vacuum (figure 1), and

- scan-coil hysteresis causing image edges to be distorted when flyback time is reduced (figure 2).

Each of these artefacts alone degrades the final image signal-to-noise ratio (SNR), precision, or fidelity, making reliable quantification far more difficult. Furthermore, when pushing towards ultra-low-dose imaging one may want to combine all the above approaches, resulting in images which are multiply compromised and potentially unreliable.

Here we present solutions to each of these issues, allowing clean, high SNR, low-dose images to be obtained. A USB-streaming oscilloscope connected to the annular dark-field (ADF) detector is used to digitise the recorded signal. From this we obtain a binary ' 1 ' each time an electron hits the detector. The digitisation localises electron hits to a single time point in the scan and records all hits with equal sensitivity, eliminating streaking and afterglow. This also satisfies the definitions of perfect modulation transfer function and detector quantum efficiency [4].

Using a single-crystal reference material with known lattice parameters we calibrate the apparent 'strain' due to the scan-coil hysteresis. For any given instrument this is reproducible for any chosen combination of image parameters (dwell-time and flyback time). This calibration allows for the correction of any future images taken with the same parameters whether crystalline or otherwise. 
To demonstrate the cross-compatibility of these techniques, results will be presented including atomicresolution digital-ADF images from gold nanoparticles using a Nion UltraSTEM 200, along with lowdose images of biological tissues taken using a ThermoFisher Titan 80-300 STEM. We will present the concept of 'useful dose-efficiency' (which may be as low as $30 \%$ when damaging flyback dose is included), before showing how this can be maximised to $\approx 95 \%$. We will present some discussion on future experiment design optimisation with respect to low-dose and low-dose-rate imaging [5].
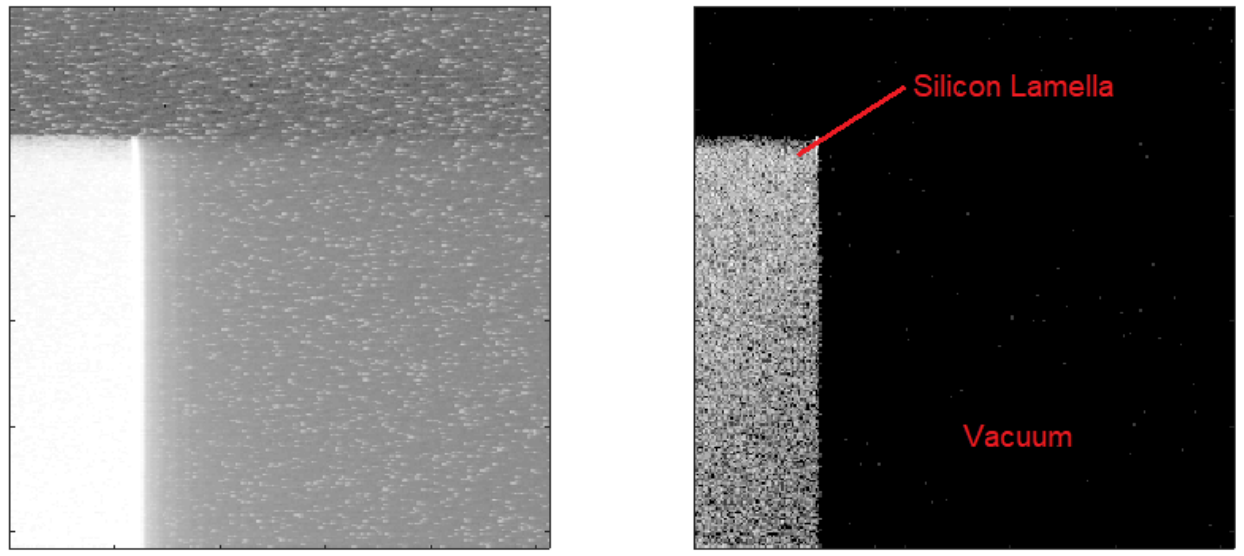

Figure 1. Example analogue (left) and digital (right) images of a silicon lamella. Individual pixel streaking as well as detector afterglow is visible in the analogue image but not in the digital one. The image gamma has been exaggerated to reveal both fast and slow contrast streaking.

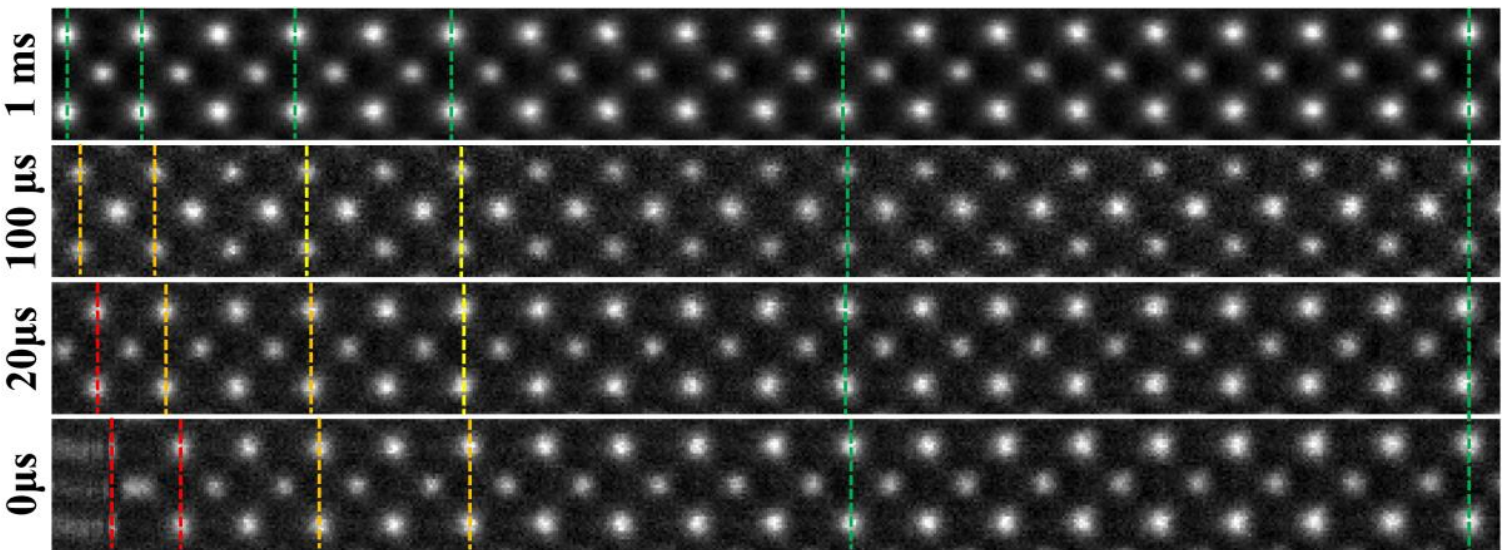

Figure 2. Montage of cropped panels from increasingly faster flyback time acquisitions of a $\mathrm{SrTiO} 3$ crystal. The image taken with $1 \mathrm{~ms}$ flyback time is used as a quasi-static reference. At faster acquisitions the unit-cells at the start of the scan lines (left of edge frames) become increasingly (but reproducibly) compressed.

References

[1] R. F. Egerton, P. Li, and M. Malac, "Radiation damage in the TEM and SEM," Micron, vol. 35, no. 6, pp. 399-409, 2004.

[2] R. F. Egerton, "Choice of operating voltage for a transmission electron microscope," Ultramicroscopy, vol. 145, pp. 85-93, 2014. 
[3] A. De Backer et al., "Dose limited reliability of quantitative annular dark field scanning transmission electron microscopy for nano-particle atom-counting," Ultramicroscopy, vol. 151, pp. 56-61, 2015.

[4] L. Jones and C. Downing, "The MTF \& DQE of Annular Dark Field STEM: Implications for Lowdose Imaging and Compressed Sensing," Microsc. Microanal., vol. 24, no. S1, pp. 478-479, 2018.

[5] Tiarnan Mullarkey acknowledges funding from the SFI CDT ACM and AMBER2, and Lewys Jones acknowledges funding from SFI and the Royal Society. 